\title{
Retinal overexpression of angiopoietin-2 mimics diabetic retinopathy and enhances vascular damages in hyperglycemia
}

\author{
Frederick Pfister $\cdot$ Yumei Wang $\cdot$ Kay Schreiter $\cdot$ Franziska vom Hagen • \\ Karin Altvater · Sigrid Hoffmann - Urban Deutsch · Hans-Peter Hammes • \\ Yuxi Feng
}

Received: 14 October 2008/ Accepted: 13 January 2009/Published online: 24 February 2009

(C) Springer-Verlag 2009

\begin{abstract}
Our previous data suggested that angiopoietin2 (Ang-2) is linked to pericyte loss, thereby playing an important role in diabetic retinopathy. In this study, we investigated the effect of retinal overexpression of human Ang-2 (mOpsinhAng2 mouse) on vascular morphology in non-diabetic and streptozotozin-induced diabetic animals. Pericyte (PC) coverage and acellular capillary (AC) formation were quantitated in retinal digest preparations after 3 and 6 months of diabetes duration. The degree of retinopathy in non-diabetic mOpsinhAng2 mice at 3 months $(-21 \%$ PC,$+49 \%$ AC) was comparable to age-matched diabetic wild type mice. Diabetic mOpsinhAng2 mice exhibited significantly worse vascular pathology than wild type counterparts at 6 months. Quantitative PCR revealed that human Ang-2 mRNA was highly overexpressed in retinas of transgenic mice. Our data demonstrate that overexpression of Ang-2 in the retina enhances vascular pathology, indicating that Ang-2 plays an essential role in diabetic vasoregression via destabilization of pericytes.
\end{abstract}

F. Pfister - Y. Wang · F. vom Hagen - K. Altvater

H.-P. Hammes · Y. Feng ( $)$

5th Medical Clinic, Medical Faculty Mannheim,

University of Heidelberg, Theodor-Kutzer-Ufer 1-3, 68167 Mannheim, Germany

e-mail: yuxi.feng@med5.ma.uni-heidelberg.de

K. Schreiter

DeveloGen AG, 37079 Göttingen, Germany

S. Hoffmann

Medical Faculty Mannheim, Medical Research Center,

University of Heidelberg, 68167 Mannheim, Germany

U. Deutsch

Theodor Kocher Institute,

University of Berne, 3012 Berne, Switzerland
Keywords Angiopoietin-2 - Diabetic retinopathy Pericyte

\section{Introduction}

Diabetic retinopathy (DR) is a common microangiopathy in diabetes. Hyperglycemia, the central initiating factor in DR, induces pathological changes in the retinal vascular bed, even early when the retina is clinically unaffected. Pericytes with glial cells enveloping capillaries are important for the protection of endothelial cells against damages from the harmful environment within the retina. In DR, hyperglycemia results in loss of pericytes, followed by degeneration of endothelial cells and capillary occlusions [2, 11, 13]. Enge et al. [3] demonstrated the association of reduced pericyte coverage with retinopathy in a transgenic mouse model with conditional inactivation of the pericyte recruiting factor PDGF-B. They found that a $50 \%$ reduction of pericytes is the threshold for the development of vascular proliferation, indicating the crucial role of pericytes in the maintenance of vascular integrity and normal function.

There is emerging evidence indicating that factors, such as the angiopoietin-Tie2 system are involved in hyperglycemia-induced pericyte loss and the progression of DR. Angiopoietin-2 (Ang-2) is an important molecule involved in the adherence of pericytes to the capillaries ensuring the maturation, integrity and physiological functions of the vasculature [5, 7,9]. Increasing evidence suggests that Ang-2 plays an important role both in physiological and in pathological angiogenesis, as well as in diabetic retinopathy $[6-8,12,15]$. Our previous data have demonstrated that Ang-2 is upregulated prior to morphological changes of retinal capillaries in diabetic retinopathy. Administration of 
Ang-2 into adult eyes results in pericyte loss. Reduction of Ang-2 in the Ang2LacZ murine retina protects capillaries from pericyte loss and decreases the numbers of acellular capillaries (ACs) in experimental diabetic retinopathy [8]. Moreover, we assessed pericyte coverage under overexpression of Ang-2 in the retinal development using a transgenic mouse line with overexpression of human Ang-2 under the mouse opsin promoter in the photoreceptor cells (mOpsinhAng2 mouse). We found reduced pericyte coverage and accelerated retinal vascular development in physiological and pathological angiogenesis in the retina demonstrating the effect of Ang-2 on capillary formation in the development [4]. However, the further fate of retinal capillaries in this gain-of-function model is unknown,

Diabetes causes Ang-2 upregulation predominantly in the ganglion cell layer and inner nuclear cell layer in the retina [12]. Endothelial and glial upregulation of Ang-2 has been evidenced $[1,10,16]$. By combining constitutive and hyperglycemia-inducible Ang-2 overexpression in the diabetic mOpsinhAng2 mice, we aimed to assess the functional consequences on retinal vessels.

\section{Materials and methods}

Animals

The care and experimental use of all animals in this study were in accordance with institutional guidelines and in compliance with the Association for Research in Vision and Ophthamology (ARVO) statement. The transgenic mOpsinhAng2 mouse was identified by genotyping as described previously [4].

\section{Diabetes induction}

Eight-week-old heterozygous mOpsinhAng2 mice and their wild type littermates were randomly divided into a non-diabetic and a diabetic group. The mice of the diabetic group were injected intraperitoneally with streptozotozin (STZ, $150 \mathrm{mg} / \mathrm{kg}$ body weight) diluted in citrate buffer $(\mathrm{pH}$ 4.5). STZ was purchased from Sigma, Germany. Diabetes was considered successfully induced as blood glucose levels were stable over $250 \mathrm{mg} / \mathrm{dl}$. Blood glucose levels and body weight were monitored regularly and $\mathrm{HbAlc}$ was measured when the mice were sacrificed at 3 and 6 months after induction of diabetes. Eyes were immediately frozen at $-80^{\circ} \mathrm{C}$ until further analysis.

RNA isolation and quantitative PCR

Retinal RNA was isolated from individual retinas homogenized in $1 \mathrm{ml}$ Trizol reagent (Invitrogen, Karlsruhe, Germany) at $4^{\circ} \mathrm{C}$ according to the manufacturer's instructions. Then, RNA was reverse transcribed with QuantiTect Reverse Transcription kit (Qiagen GmbH, Germany) and subjected to Taqman analysis using the Taqman $2 \times$ PCR master Mix (Applied Biosystems, Weiterstadt, Germany). The expression of genes was analysed by the $2^{-\Delta \Delta \mathrm{CT}}$ method using $18 \mathrm{~s}$ ribosomal RNA as housekeeping control. All primers were designed according to the specific sequences in the Genbank and purchased from IBA (Göttingen, Germany) and primers and FAM-labeled Taqman TAMRA probes used were: S18: forward 5'-ACC ACA TCC AAG GAA GGC AG-3', reverse 5'-TTT TCG TCA CTA CCT CCC C-3' ${ }^{\prime}$, probe 5'-AGG CGC GCA AAT TAC CCA CTC CC-3'; VEGF: forward 5'-CGA GAT AGA GTA CAT CTT CAA GCC G-3', reverse $5^{\prime}$-TCA TCG TTA CAG CAG CCT GC-3' ${ }^{\prime}$, probe $5^{\prime}$-CTG TGT GCC GCT GAT GCG CTG-3'; Ang-1: forward 5'-CAA CAA CAG CAT CCT GCA GAA- ${ }^{\prime}$, reverse $5^{\prime}$-CTT TAG TGC AAA GGC TGA TAA GGT T- $3^{\prime}$, probe $5^{\prime}$-CAA CAA CTG GAG CTC ATG GAC ACA GTT CA-3'; mAng2: forward 5'-TGC GGA AAT CTT CAA GTC AGG- $3^{\prime}$, reverse $5^{\prime}$-CCT TGA TCT CCT CTG TGG AGT TG-3', probe 5'-CAC CAG TGG CAT CTA CAC ACT GAC CTT CC-3'; hAng2: forward 5'-TCC TCC TGC CAG AGA TGG AC-3', reverse 5'-TGC ACA GCA TTG GAC ACG TA-3', probe 5'-ACT GCC GCT CTT CCT CCA GCC C-3'; Tie2: forward 5'-ACA TAG GAG GAA ACC TGT TCA CCT-3', reverse 5'-CCC CCA CTT CTG AGC TTC AC-3', probe 5'-AGC CTT CAC CAG GCT GAT TGT TCG G-3'.

\section{Retinal digest preparation}

Retinas of mOpsinhAng2 mice were fixed in $4 \%$ paraformaldehyde for 24-48 h. Then, retinas were incubated in aqua bidest for $30 \mathrm{~min}$. Subsequently, the retinas were transferred to a $3 \%$ trypsin solution resolved in $0.2 \mathrm{M}$ Tris buffer, $\mathrm{pH} 7.4$, for 2-3 h. The retinas of mOpsinhAng2 mice were washed with aqua bidest, and isolated retinal vasculature was flat mounted on objective slides. For quantification of pericyte numbers, the samples were stained with periodic acid Schiff base and hematoxylin (PASH). During digestion, vitreous with inner limiting membrane were carefully moved away.

Quantitative retinal morphometry

Analysis of pericyte coverage of the retinal vasculature was carried out in the retinal digest preparations stained with PASH as described previously [8]. In brief, the total numbers of pericytes were counted in ten randomly selected fields of the retina using an image analyzing system (CUE-2; Olympus Opticals, Hamburg, Germany), and their numbers were normalized to the relative capillary density (numbers of cells per $\mathrm{mm}^{2}$ of capillary area). The 
numbers of ACs were counted according to an established method in ten randomly selected fields as described previously [8]. Samples were evaluated in a masked fashion.

\section{Statistic analysis}

Data are expressed as mean $\pm \mathrm{SD}$. Data analysis was performed by using ANOVA and a $p<0.05$ was considered as statistical significance.

\section{Results}

Overexpression of Ang-2 in the retina does not change metabolic parameters in experimental diabetic retinopathy

At 6 months, non-diabetic mOpsinhAng2 mice showed normal blood glucose levels and similar body weight compared to the non-diabetic wild type mice. Blood glucose was $178 \pm 23 \mathrm{mg} / \mathrm{dl}$ in non-diabetic mOpsinhAng2 mice and $164 \pm 18 \mathrm{mg} / \mathrm{dl}$ in non-diabetic wild type mice, while body weight was $35 \pm 5 \mathrm{~g}$ in non-diabetic mOpsinhAng2 mice and $36 \pm 5 \mathrm{~g}$ in non-diabetic wild type mice. Overexpression of Ang-2 did not change HbAlc values in transgenic mice (mOpsinhAng2 $5.4 \pm 0.9 \%$ vs. wild type $5.2 \pm 1.1 \%$ ). As in wild type animals, streptozotozin injection induced significantly hyperglycemia, body weight loss and elevated HbA1c in mOpsinhAng2 mice. Diabetic mOpsinhAng2 mice had blood glucose levels comparable to diabetic wild type mice (diabetic mOpsinhAng2: $587 \pm 33 \mathrm{mg} / \mathrm{dl}$, diabetic wild type mice: $593 \pm 17 \mathrm{mg} / \mathrm{dl}$ ). Body weight of $23 \pm 5 \mathrm{~g}$ in diabetic mOpsinhAng2 mice was similar to $25 \pm 3 \mathrm{~g}$ in diabetic wild type mice. Elevated HbA1c did not differ significantly in diabetic mOpsinhAng2 and diabetic wild type mice.

Overexpression of Ang-2 in the retina mimics DR and aggravates vascular damages in experimental diabetic retinopathy

To assess the relationship between retinal overexpression of Ang- 2 and pericyte coverage in hyperglycemia, we analysed pericyte coverage of heterozygous mOpsinhAng2 retinas after 3- and 6-month of hyperglycemia using retinal digest preparations. Overexpression of Ang-2 in the non-diabetic retina led to significant pericyte dropout of about $21 \%$ compared to the non-diabetic wild type retina at 3 and 6 months $\left(1,705 \pm 155 / \mathrm{mm}^{2}\right.$ of capillary area in nondiabetic mOpsinhAng2 mice vs. $2,161 \pm 144 / \mathrm{mm}^{2}$ of capillary area in non-diabetic wild type mice at 3 months, $1,720 \pm 68 / \mathrm{mm}^{2}$ of capillary area in non-diabetic mOpsinhAng2 mice vs. $2,151 \pm 109 / \mathrm{mm}^{2}$ of capillary area in non-diabetic wild type mice at 6 months, respectively.
Fig. 1 Vascular morphological demonstration of diabetic mOpsinhAng2 retinas.

Representative demonstration (a-d) of retinal vascular morphology in retinal digest preparations. a, b wild type mice; c, $\mathbf{d}$ mOpsinhAng2 mice; a, c 6 months non-diabetic mice; b, d 6 months diabetic mice. Arrows: acellular capillaries. Magnifications: $400 \times$

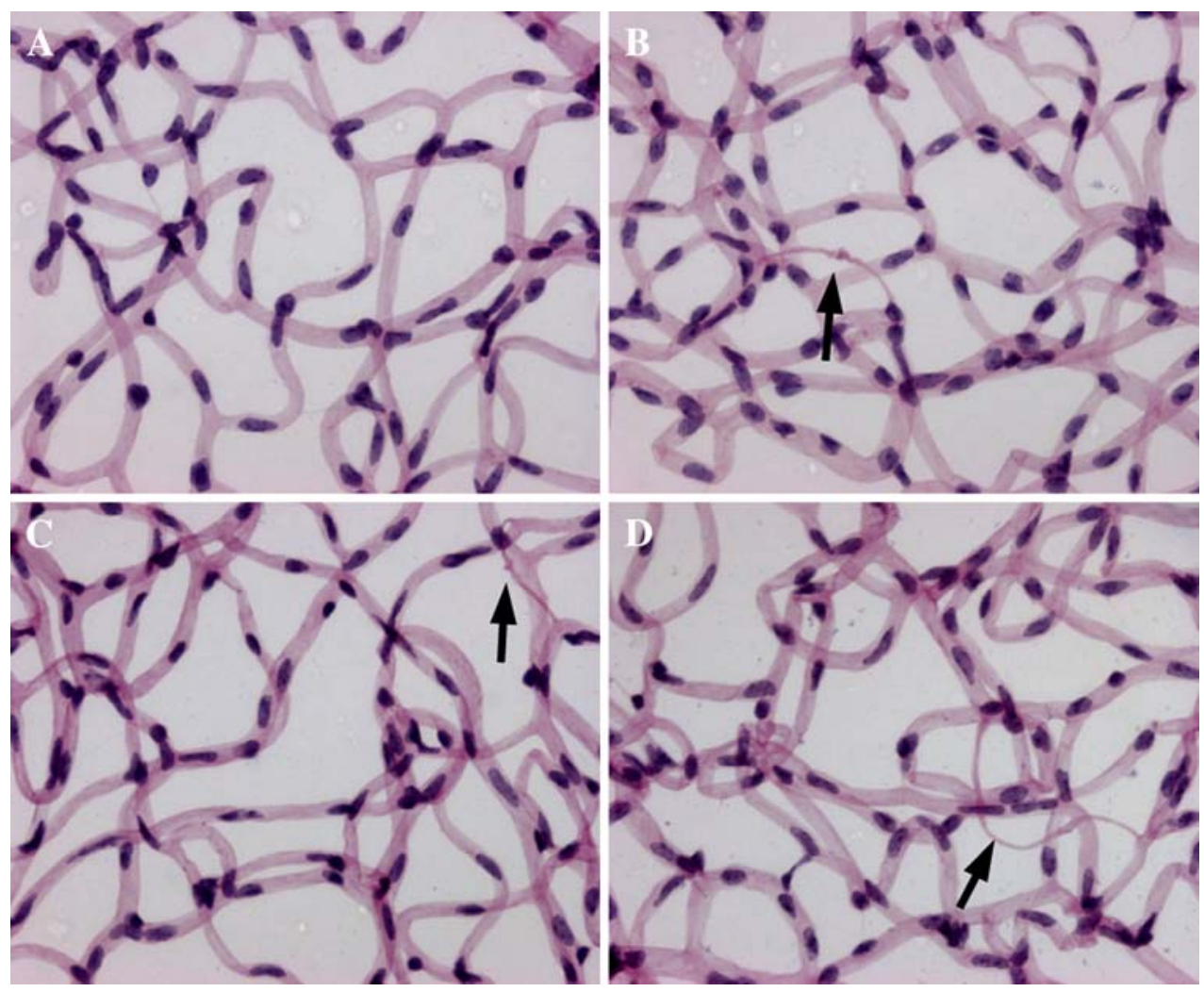




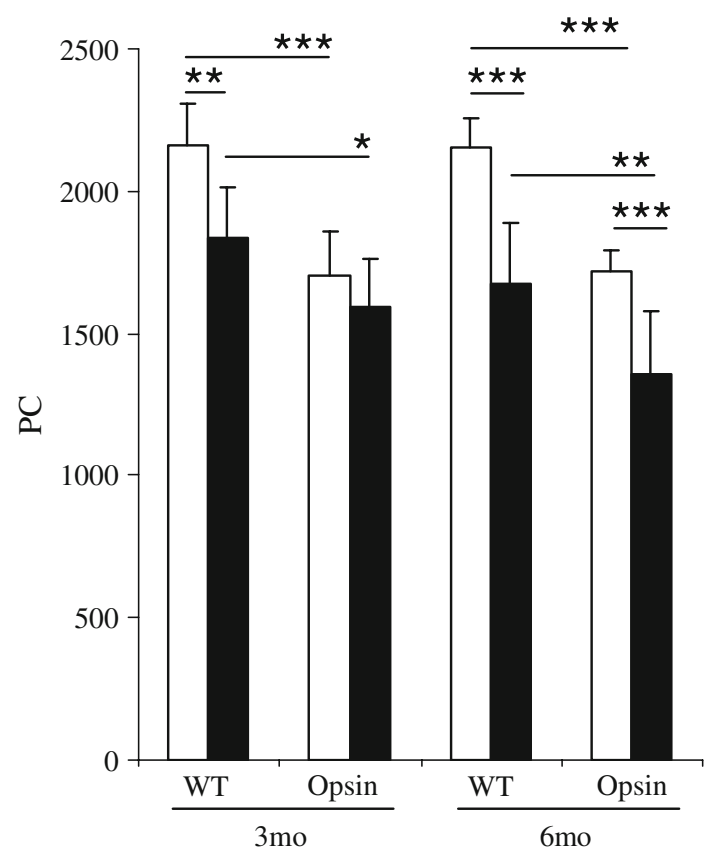

Fig. 2 Pericyte coverage of diabetic mOpsinhAng2 retinas. Pericyte coverage was quantitatively analysed in retinal digest preparations. Opsin: mOpsinhAng2, and WT: wild type mice in diabetic (filled square) and non-diabetic (open square) mice at 3 and 6 months after diabetes induction. $n=6$. Data are means \pm SD. $* p<0.05$, $* * p<0.01, * * * p<0.001$

Figs. 1, 2). A significant decrease of pericyte numbers was observed in diabetic wild type mice at 3 and 6 months ( $p<0.01$ and $p<0.001$ vs. non-diabetic wild type mice, respectively). Pericyte reduction in non-diabetic mOpsinhAng2 mice at 3 and 6 months were similar to the reduced pericyte numbers of age-matched diabetic wild type mice. Hyperglycemia had no influence on pericyte numbers at 3 months in mOpsinhAng2 mice ( $p>0.05)$, but diabetes in mOpsinhAng2 mice led to more pericyte loss in the retina at 6 months $\left(1,355 \pm 226 / \mathrm{mm}^{2}\right.$ of capillary area in diabetic mOpsinhAng2 mice and $1,671 \pm 220 / \mathrm{mm}^{2}$ of capillary area in diabetic wild type mice, Figs. 1, 2, $p<0.001$ ).

Consequently, diabetes-induced formation of ACs was determined in the retinas of mOpsinhAng2 mice. Hyperglycemia increased the formation of ACs in wild type mice after 6 months of diabetes duration, as demonstrated in Figs. 1 and 3. At 3 months, overexpression of Ang-2 in the retina caused increased ACs in non-diabetic animals $\left(29 \pm 5 \mathrm{AC} / \mathrm{mm}^{2}\right.$ of retinal area in non-diabetic mOpsinhAng2 mice vs. $20 \pm 5 \mathrm{AC} / \mathrm{mm}^{2}$ of retinal area nondiabetic wild type mice), similar to increased ACs in diabetic wild type mice $\left(27 \pm 6 \mathrm{AC} / \mathrm{mm}^{2}\right.$ of retinal area). At 6 months, formation of ACs remained elevated in nondiabetic mOpsinhAng2 mice. Diabetes shifted the formation of ACs in transgenic mice in similar extent to diabetic wild type mice and thereby onto the highest level among the age-matched groups $\left(40 \pm 7 \mathrm{AC} / \mathrm{mm}^{2}\right.$ of retinal area in

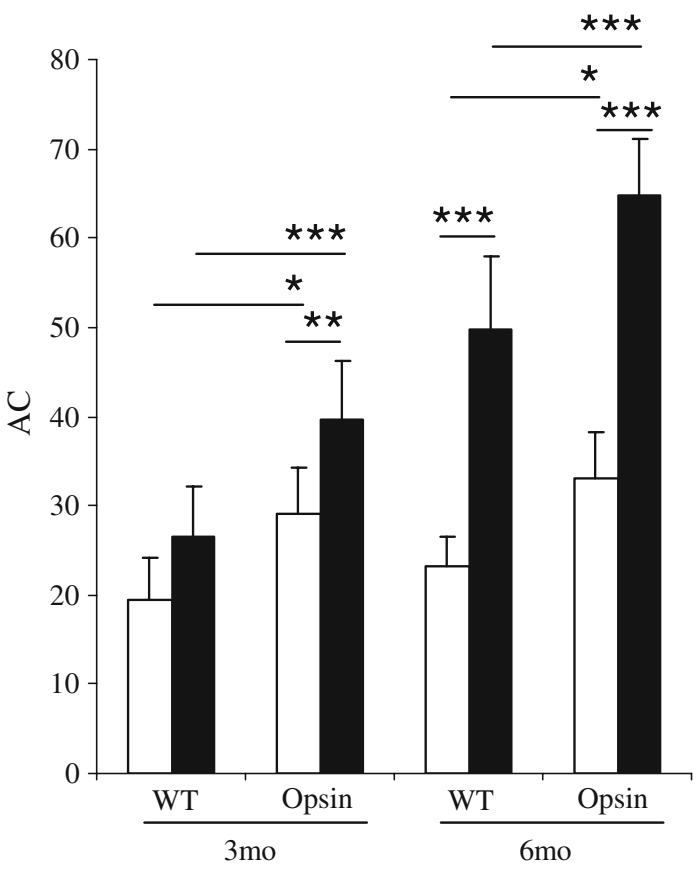

Fig. 3 Formation of acellular capillaries of diabetic mOpsinhAng2 retinas. Segments of acellular capillaries were quantitatively analysed in retinal digest preparations. Opsin: mOpsinhAng2, and WT: wild type mice in diabetic (filled square) and non-diabetic (open square) mice at 3 and 6 months after diabetes induction. $n=6$. Data are mean \pm SD. $* p<0.05, * * p<0.01, * * * p<0.001$

diabetic mOpsinhAng2 vs. $29 \pm 5 \mathrm{AC} / \mathrm{mm}^{2}$ of retinal area in non-diabetic mOpsinhAng2 mice at 3 months, $65 \pm 6 \mathrm{AC} / \mathrm{mm}^{2}$ of retinal area in diabetic mOpsinhAng2 vs. $33 \pm 5 \mathrm{AC} / \mathrm{mm}^{2}$ of retinal area in non-diabetic mOpsinhAng2 mice at 6 months). No retinal vascular abnormalities such as microaneurysms and dilatation of vessels were observed in retinal digest preparations of mOpsinhAng2 mice (data not shown).

Human Ang-2 is highly overexpressed in transgenic retinas

Variation in gene expression precedes vascular morphological changes in the 3- and 6- month retinas; therefore, we performed quantitative real time PCR using 3-week retinas to identify the gene expression of endogenous Ang2, Ang-1 Tie2 and VEGF, and transgenic human Ang-2. Human Ang-2 was strongly overexpressed in transgenic retinas, whereas it was not detectable in retinas of wild type mice. Retinal overexpression of human Ang-2 in non-diabetic transgenic animals did not affect the expression of endogenous Ang-1, mouse Ang-2 and their receptor Tie2 compared to non-diabetic wild type mice. Morover, 3-week hyperglycemia, both in transgenic and non-transgenic animals did not alter the expression of Ang-1, mouse and human Ang-2 and their receptor Tie2 compared to their 


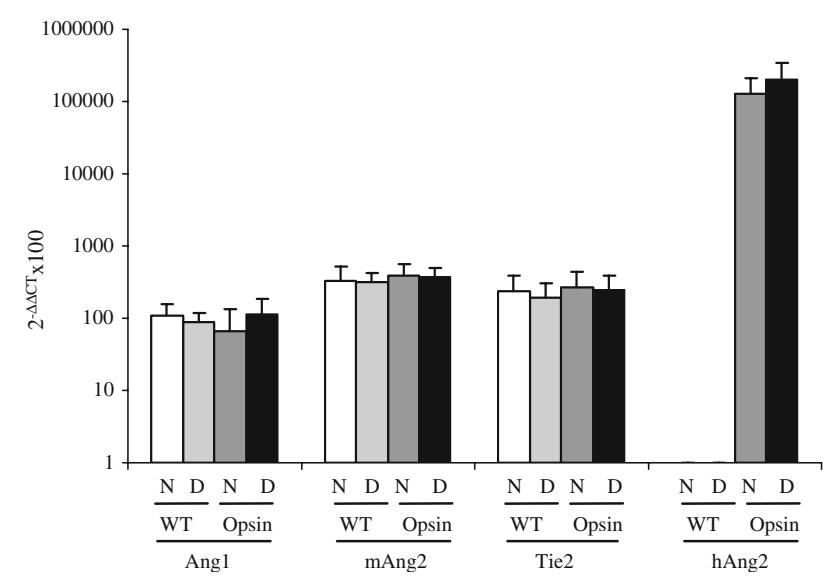

Fig. 4 Expression of Ang-1, mouse and human Ang-2, and Tie2 in diabetic mOpsinhAng2 retinas at 3 weeks after diabetes induction. $N$ non-diabetic, $D$ diabetic, Opsin mOpsinhAng2 mice, $W T$ wild type mice. mAng2 mouse angiopoietin-2, hAng2 human angiopoietin-2. $n=5-9$. Expression of Ang-1 in non-diabetic wild type is set as 100 because of logarithmic scales on the $y$ axis. Data are mean \pm SD

non-diabetic controls (Fig. 4). Modulation of VEGF expression was observed neither in transgenic mOpsinhAng2 retinas compared with their non-transgenic animals, nor in 3-week hyperglycemic non-transgenic and transgenic retinas compared with their non-diabetic controls (data not shown).

\section{Discussion}

Our study demonstrates that constitutive overexpression of Ang-2 in the retinal photoreceptor cells induces vascular pathology, mimicking diabetic retinopathy. Furthermore, we show that constitutive overexpression of Ang-2 enhances further vascular damages in hyperglycemia.

Retinal vasoregression is characteristic for DR. We observed that overexpression of Ang-2 in retinal neurons leads to pericyte loss and $\mathrm{AC}$ formation in the normoglycemic retina. The retinal vascular changes of normoglycemic mOpsinhAng2 mice resemble qualitatively and quantitatively those observed in 3 month diabetic wild type mice. Together with our previous data showing pericyte loss is linked to augmented Ang-2 from vitreous and glia [8], the data in present study reinforce the importance of Ang-2 in controlling pericyte coverage of mature retinal vessels and its crucial role in the development of DR.

Compared with development of pericyte loss in diabetic wild type mice, hyperglycemia did not result in a further extent of pericyte loss in diabetic mOpsinhAng2 retinas at 3 months. The lack of progression in pericyte loss in diabetic mOpsinhAng2 retinas suggests that there might be a temporal validity of Ang-2 or a threshold for pericyte loss in mOpsinhAng2 retinas. Enge et al. [3] found a 50\% reduction of PCs is the threshold for the development of proliferative DR correlated with PDGF-B. Similarly, we found that there likely is a threshold level of approximately $20 \%$ pericyte loss for Ang-2 dependent vasoregression. Nevertheless, this threshold was broken by long-term hyperglycemia of 6 months. In the combined model, hyperglycemia induces an additional release of Ang- 2 from glial cells, explaining the further increase of pericyte loss and vasoregression in diabetic mOpsinhAng2 mice at 6 months. Other hyperglycemia-induced factors within the retina, which additively promote retinal vasoregression could participate in the further increase of retinal pericyte loss and AC formation in diabetic mOpsinhAng2 retinas.

In the normal retina, Ang-2 is expressed in neuronal cells and in some undefined cell types of the ganglion cell layer [6]. In the diabetic retina, endothelial cells and glial cells might be the main sources of Ang- $2[1,10,16]$. An increase of Ang-2 expression has been described in the inner nuclear layer after 6 months of hyperglycemia [12]. However, the precise mechanisms underlying Ang-2 upregulation in the diabetic retina remain to be elucidated. Recently, Yao et al. [16] reported that hyperglycemia induces intracellular formation of methylglyoxal which activates Ang-2 transcription through modification of $\mathrm{mSin} 3 \mathrm{~A}$. In a parallel experiment using the same mOpsinhAng2 mice, we showed that overexpression of Ang-2 results in elevated pericyte migration in certain pericyte subpopulations, indicating a novel mechanism for pericyte loss in DR [14]. In the present study, we quantitatively demonstrate that overexpression of Ang-2 results in significant pericyte loss and formation of ACs comparable to diabetic wild type mice. Thus, mOpsinhAng2 mouse presents a suitable mouse model for elucidating mechanisms involved in retinal vasoregression related to elevated Ang2 levels. In the present study, we did not observe an increase in RNA expression of Ang-2 in diabetic wild type mice in contrast to what we reported before in the rat [8]. Species difference may be one explanation, and the other could be the early time point studied ( 3 weeks), as vascular damage in the mouse retina is generally milder than in the rat. In comparison with our previous observation, a $20 \%$ decrease of pericyte coverage in diabetic mouse retina at 6 months has been revealed in diabetic rat retina at 3 months after diabetes induction [8]. In this study, we provide evidence that human Ang-2 is highly expressed in transgenic retinas, resulting in earlier vascular pathology in the retina.

In conclusion, our study provides evidence that Ang-2 plays a critical role in retinal vascular maintenance and in retinal vascular damage of early diabetic retinopathy. These data contribute to the understanding of Ang-2 function in retinal vasoregression and thus are important 
for development of therapeutic interventions to prevent diabetic retinopathy.

Acknowledgments This study was supported by grants from the DFG, the DDG and the GRK 880. The authors thank Petra Bugert, Nadine Dietrich and Valerie Schwarz for their grateful support.

\section{References}

1. Chi JT, Chang HY, Haraldsen G et al (2003) Endothelial cell diversity revealed by global expression profiling. Proc Natl Acad Sci USA 100:10623-10628

2. Cogan DG, Toussaint D, Kuwabara T (1961) Retinal vascular patterns. IV. Diabetic retinopathy. Arch Ophthalmol 66:366-378

3. Enge M, Bjarnegard M, Gerhardt $\mathrm{H}$ et al (2002) Endotheliumspecific platelet-derived growth factor-B ablation mimics diabetic retinopathy. EMBO J 21:4307-4316

4. Feng Y, vom Hagen F, Pfister F et al (2007) Impaired pericyte recruitment and abnormal retinal angiogenesis as a result of angiopoietin-2 overexpression. Thromb Haemost 97:99-108

5. Gale NW, Thurston G, Hackett SF et al (2002) Angiopoietin-2 is required for postnatal angiogenesis and lymphatic patterning, and only the latter role is rescued by Angiopoietin-1. Dev Cell 3:411423

6. Hackett SF, Ozaki H, Strauss RW et al (2000) Angiopoietin 2 expression in the retina: upregulation during physiologic and pathologic neovascularization. J Cell Physiol 184:275-284

7. Hackett SF, Wiegand S, Yancopoulos G et al (2002) Angiopoietin-2 plays an important role in retinal angiogenesis. J Cell Physiol 192:182-187
8. Hammes HP, Lin J, Wagner P et al (2004) Angiopoietin-2 causes pericyte dropout in the normal retina: evidence for involvement in diabetic retinopathy. Diabetes 53:1104-1110

9. Maisonpierre PC, Suri C, Jones PF et al (1997) Angiopoietin-2, a natural antagonist for Tie2 that disrupts in vivo angiogenesis. Science 277:55-60

10. Matsumara T, Hammes HP, Thornalley P et al. (2000) Hyperglycemia increases angiopoietin-2 expression in retinal Muller cells through through superoxide-induced overproduction of a-oxoaldehyde age precursors (abstract). Diabetes 49: A55

11. Ochs M, Mayhew TM, Knabe W (2000) To what extent are the retinal capillaries ensheathed by Muller cells? A stereological study in the tree shrew Tupaia belangeri. J Anat 196(Pt 3):453461

12. Ohashi H, Takagi H, Koyama $\mathrm{S}$ et al (2004) Alterations in expression of angiopoietins and the Tie- 2 receptor in the retina of streptozotocin induced diabetic rats. Mol Vis 10:608-617

13. Orlidge A, D'Amore PA (1987) Inhibition of capillary endothelial cell growth by pericytes and smooth muscle cells. J Cell Biol 105:1455-1462

14. Pfister F, Feng Y, vom Hagen F et al (2008) Pericyte migration: a novel mechanism of pericyte loss in experimental diabetic retinopathy. Diabetes 57:2495-2502

15. Watanabe D, Suzuma K, Suzuma I et al (2005) Vitreous levels of angiopoietin 2 and vascular endothelial growth factor in patients with proliferative diabetic retinopathy. Am J Ophthalmol 139:476-481

16. Yao D, Taguchi T, Matsumura $T$ et al (2006) Methylglyoxal modification of mSin3A links glycolysis to angiopoietin-2 transcription. Cell 124:275-286 